Bayero Journal of Pure and Applied Sciences, 11(1): 377 - 380

ISSN 2006 - 6996

\title{
ON CERTAIN COMBINATORIAL PROBLEMS OF THE SEMIGROUP OF PARTIAL AND FULL CONTRACTIONS OF A FINITE CHAIN
}

\author{
Zubairu, ${ }^{1}$ M. M. and Bashir $\mathrm{Ali}^{2}$ \\ ${ }^{1}$ Department of Mathematics, Bayero University Kano, PM. Box 3011 Kano Nigeria \\ ${ }^{2}$ Department of Mathematics, Nigeria Defence academy \\ 'mmzubairu.mth@buk.edu.ng; ${ }^{2}$ bali@nda.edu.ng
}

\section{ABSTRACT}

Denote $[n]=\{1,2, \ldots, n\}$ to be a finite $n$-chain, where $n$ is a natural number. Let $P_{n}$ and $T_{n}$ denote the semigroups of partial and full transformations of $[n]$, respectively. Let $C_{P_{n}}=$ $\left\{\alpha \in P_{n}:|x \alpha-y \alpha| \leq|x-y| \forall x, y \in D o m \alpha\right\}$ and $C T_{n}=\left\{\alpha \in T_{n}:|x \alpha-y \alpha| \leq|x-y| \forall x, y \in[n]\right\}$, then $C P_{n}$ and $C T_{n}$ are known to be subsemigroup of $P_{n}$ and $T_{n}$, respectively. The algebraic properties of these semigroup have been investigated, however the combinatorial properties are yet to be investigated. In this paper, combinatorial problems (or questions) of these subsemigroups where explored. Let $D C P_{n}=\left\{\alpha \in D P_{n}:|x \alpha-y \alpha| \leq|x-y| \forall x, y \in D o m \alpha\right\}$ and $D C T_{n}=\left\{\alpha \in D T_{n}:|x \alpha-y \alpha| \leq|x-y| \forall x, y \in[n]\right\}$ (where $D P_{n}$ and $D T_{n}$ are the semigroup of order decreasing partial and full transformations, respectively. ) Then $D_{C C P_{n}}$ and $D_{C C T_{n}}$ are known to be the semigroup of order decreasing partial and full contractions, respectively. In this paper we give a necessary and sufficient conditions for an element to be regular for the semigroups $\mathrm{DCP}_{n}$ and $\mathrm{DCT}_{n}$.

Keywords: Transformation semigroup, Contractions, Number of fixed point, equivalences

INTRODUCTION

Let $[n]=\{1,2, \ldots, n\}$ be a finite chain. A map which has its domain and range both subset of $[n]$ is said to be a transformation of $[n]$. Denote $\operatorname{Dom} \alpha$ and $\operatorname{Im} \alpha$ to be the domain and image of a transformation $\alpha$, respectively. If $\operatorname{Dom} \alpha \subseteq$ $[n]$ then $\alpha$ is said to be a partial transformation and if $\operatorname{Dom} \alpha=[n]$ then $\alpha$ is said to be a full transformation. The collection of all partial (resp., full) transformation is known to be the semigroup of partial(resp., full) transformation of a finite chain. We adopt as in the literature, the notations $P_{n}$ and $T_{n}$ to be the semigroups of partial and full transformations of $[n]$. Algebraic and combinatorial properties of these semigroup where extensively studied in the literature, for example see Preston, Mazorchuk and Howie (Clifford and Preston, 1961; Ganyushkin and Mazorchuk, 2009; Howie, 1995). For proper understanding of the concept of semigroup, we refer the reader to any of Preston, Mazorchuk and Howie (Clifford and Preston, 1961; Ganyushkin and Mazorchuk, 2009; Howie, 1995) as well.

Let $\quad C P_{n}=\left\{\alpha \in P_{n}:|x \alpha-y \alpha| \leq|x-y| \forall x, y \in\right.$ Dom $\alpha\}$ and $C T_{n}=\left\{\alpha \in T_{n}:|x \alpha-y \alpha| \leq \mid x-\right.$ $y \mid \forall x, y \in[n]\}$, then $C P_{n}$ and $C T_{n}$ are known to be subsemigroup of $P_{n}$ and $T_{n}$, respectively. The study of these semigroups and their respective subsemigroups was first initiated in 2012 by Umar and Alkharousi (2012) supported by a grant from The Research Council of Oman (TRC). In the proposal by Umar and Alkharousi (2012), notations for the semigroups and their subsemigroups were given, as such we maintain the same notations throughout this paper. The algebraic study of the semigroup $C T_{n}$ was initiated by Garba et al. (2017), where they successfully characterized its starred Green's equivalences and later Umar and Zubairu (2018) shows that this semigroup is left abundant and give a complete characterization of its Green's equivalences. The algebraic study of the semigroup of partial contractions was initiated by Ali et al. (2018), they characterizes the Green's equivalences and shows that this semigroup and some of its subsemigroup is not regular. They equally give a necessary and sufficient condition for an element to be regular in the semigroup. In the same year Umar and Zubairu (2018) shows that the semigroup of partial contractions $C P_{n}$ is left abundant.

It is natural to ask combinatorial questions from any given transformation semigroup, as these semigroups on a finite chain are finite, and there are allot of equivalences which usually arises from the algebraic studies of any transformation semigroup. This includes its Green's equivalences and the rest. Usually answer to these combinatorial questions solves the problem of finding the order of these semigroups, otherwise they gives some fascinating numbers or triangle of numbers or sequences, some which are already recorded in the Online encyclopedia of integer sequences or they form some new sequences. 
Special Conference Edition, November, 2018

To begin our investigations let us give the definition of some basic term and their illustrations. Let $\alpha$ be an element of a semigroup $S \in\left\{P_{n}, T_{n}, C P_{n}, C T_{n}\right\}$, the width(or breadth) and the height of $\alpha$ usually denoted as $h(\alpha)$ and $b(\alpha)$, respectively is defined to be $|\operatorname{im} \alpha|$ and $|\operatorname{Dom} \alpha|$, respectively. An element $x \in \operatorname{Dom} \alpha$ is said to be a fixed point of $\alpha$ if $x \alpha=x$. The collection of all fixed point of $\alpha$ is denoted as Fix $\alpha=\{x \in \operatorname{Dom} \alpha: x \alpha=x\}$ and the order of this set is denoted by $f(\alpha)=\mid$ Fix $\alpha \mid$. The right (resp., left) waist of $\alpha$ is $w^{+}=$ $\max (\operatorname{im} \alpha) \quad$ (resp., $\left.\quad w^{-}=\min (\operatorname{im} \alpha)\right)$. The collapse of $\alpha$ is $c(\alpha)=\mid \cup_{t \in i m \alpha}\left\{t \alpha^{-1}:\left|t \alpha^{-1}\right| \geq\right.$ 2\}|.

For purpose of illustrations, consider $\alpha=$ $\left(\begin{array}{lll}1 & 2 & 3 \\ 2 & 3 & 3\end{array}\right)$. Then $\alpha=\{2,3\}$, Dom $\alpha=\{1,2,3\}$, Fix $\alpha=\{3\}$, the right waist of $\alpha$ is 3 and the left waist of $\alpha$ is 2 . Therefore $h(\alpha)=2$, $b(\alpha)=3$ and $f(\alpha)=1$.

Now let $F(n ; r, q, p, m, k)=\mid\{\alpha \in S: \Lambda(b(\alpha)=$ $\left.\left.r, c(\alpha)=q, h(\alpha)=p, f(\alpha)=m, w^{+}(\alpha)=k\right)\right\} \mid$.

Denote $L=\{r, q, p, m, k\}$ to be the set of counters for the breadth, collapse, right waist, height and fix of $\alpha$. As such any five parameter combinatorial function can be express as $f\left(n ; x_{1}, x_{2}, x_{3}, x_{4}, x_{5}\right)$. Where $\left\{x_{1}, x_{2}, x_{3}, x_{4}, x_{5}\right\} \subseteq$ $L$. Similarly, we can have a combination of lower number of parameters, e. g., $F(n ; r, q, p, m), F(n ; r, q, p), F(n ; r), \quad F(n ; m)$, $F(n ; r, p), F(n ; p)$, e. t. c. It is well known that $|S|=\sum_{r} F(n ; r)=\sum_{p} F(n ; p)=\sum_{m} F(n ; m)=$ $p F(n ; r, p)$ and any 3-parameter function can be expressed as a sum of appropriate 4-parameter function and so on.

In an ideal situation we will like to compute the function $F(n ; r, q, p, m, k)$ for any finite transformation semigroup, however the task is a difficult one. For the smaller functions like $F(n ; r), \quad F(n ; m), \quad F(n ; r, p), \quad F(n ; p)$, some milestones have been achieved so far for the semigroups $P_{n}$ and $T_{n}$. For most of these combinatorial results for the two semigroups $P_{n}$ and $T_{n}$, we refer the reader to Umar (2014).

In this paper we shall give the existing results for the 2 parameter functions for the semigroups $P_{n}$ and $T_{n}$ which are all recorded in Umar (2014). We also ask similar questions for the new semigroups $C P_{n}$ and $C T_{n}$. These combinatorial problems are yet to be investigated.

A transformation $\alpha \in C P_{n}$ (resp., $\alpha \in C T_{n}$ ) is said to be order decreasing if $x \alpha \leq x$ for all $x \in \operatorname{Dom} \alpha$ (resp., $x \in[n]$ ). The collection of all order decreasing partial (resp., full) contractions is known to be the semigroup of order decreasing partial (resp., full) contractions, $D C P_{n}$ and $D C T_{n}$, respectively. In this paper we have shown that $F(n, r)=1$ for $r=1$ and $n$ and give a conjecture for $F(n, 2)$ and $F(n, 3)$ for the semigroup, $D C T_{n}$. However, the question for $F(n, r)(r \geq 4)$ remain open problem.

In this paragraph, we briefly described the structure of this paper. Section one of the paper gives the introduction, definition of some basic terms as well as the literature review. Further, questions raised in this paper has been introduced. In section 2 and 3 , combinatorial properties of the semigroups $P_{n}$ and $T_{n}$ are given. Questions for the combinatorial properties for the new semigroups $C P_{n}$ and $C T_{n}$ were explored. In section 4 , we give a neccessary and sufficient condition for an element to be regular for the semigroup $D C T_{n}$. We further give $F(n ; r)$ (for $r=1, n)$ and give a conjuncture for $F(n ; r)$ (for $r=2,3$ ) for the semigroup of order decreasing full contractions, $D C T_{n}$.

The semigroup of Partial transformations

Recall that $\alpha$ is said to be a partial transformation if Dom $\alpha \subseteq[n]$ and the collection of all partial transformations of [n] is known to be the semigroup of partial transformation usually denoted by $P_{n}$. This semigroup has been extensively studied in the literature. In this section we itemize some of the well know combinatorial properties of this semigroup and we ask a similar question for its subsemigroup of partial contractions $C P_{n}$. The Stirling number of the second kind usually denoted as $S(n, k)$ is the number of partition of the set $[\mathrm{n}]$ into exactly $\mathrm{k}$ parts $(1 \leq k \leq n)$. We begin with the following lemmas.

Lemma 2.1([12], Corollary 2.3) Let $=P_{n}$. Then $F(n ; r)=\left(\begin{array}{l}n \\ r\end{array}\right) n^{r},(n \geq r \geq 0)$.

Lemma2.2 ([6], Corollary 2) Let $S=P_{n}$. Then $F(n ; p)=\left(\begin{array}{l}n \\ p\end{array}\right) S(n+1, p+1) p !,(n \geq p \geq 0)$.

Lemma2.3([12], Corollary 2.6) Let $S=P_{n}$. Then $F(n ; m)=\left(\begin{array}{c}n \\ m\end{array}\right) n^{n-m},(n \geq m \geq 0)$.

Lemma 2.4 ([12], Corollary 2.9) Let $=P_{n}$. Then $F(n ; k)=(k+1)^{n}-k^{n},(n \geq k \geq 1)$.

We pause here to elaborate on the above lemmas, these four lemmas have solve the problems of all 2-parameter functions for the semigroup of partial transformations $P_{n}$. And summation over any of the parameter gives the order of the semigroup. For example the order of $P_{n}$ is known to be $(n+1)^{n}$ and notice that using Lemma 2.1 we have; 


$$
\begin{aligned}
\sum_{r=0}^{n} F(n, r) & =F(n ; 0)+F(n ; 1)+ \\
F(n ; 2)+F(n ; 3)+\cdots+ & F(n, n) \\
& =\left(\begin{array}{c}
n \\
0
\end{array}\right)+\left(\begin{array}{l}
n \\
1
\end{array}\right) n+ \\
\left(\begin{array}{l}
n \\
2
\end{array}\right) n^{2}+\ldots+\left(\begin{array}{l}
n \\
n
\end{array}\right) n^{n}= & (1+n)^{n} .
\end{aligned}
$$

Thus, finding these functions leads to the solution of problem of finding the order of the semigroup. In view of the above discussions we ask the following question:

Question (1) What is $(n ; r), F(n ; p), \quad F(n ; m)$ and $F(n ; k)$ when we replace $P_{n}$ with $C P_{n}$ ? These questions remain an open problem, in fact the order of the semigroup of partial contractions of a finite chain is not known yet.

An element $\varepsilon$ in a semigroup $S$ is said to be an idempotent if $\varepsilon^{2}=\varepsilon$, the collection of all idempotent of $S$ is usually denoted by $E(S)$. Idempotents of a transformation semigroup were characterized as follows; an element $\varepsilon$ in a transformation semigroup is an idempotent if and only if the blocks of $\varepsilon$ are stationary if and only if Fix $\varepsilon=i m \varepsilon$. Order of set of idempotent of various class of semigroup of transformation have been found and recorded in Umar (2014). For example if $S=P_{n}$ then the number of idempotent in $S$ is given as:

$$
|E(S)|=\sum_{r=0}^{n}\left(\begin{array}{l}
n \\
r
\end{array}\right)(r+1)^{n-r} .
$$

This result was found in Garba [6].

Question(2) Now what if $S=C P_{n}$ ? What is $|E(S)|$ ? This remain an open problem.

An element $\varepsilon$ in a semigroup $S$ is said to be a nilpotent if $\varepsilon^{k}=0$ (for some positive integer $k$ ), where 0 is referred to as empty chart or empty transformation. The collection of all nilpotent of $S$ is denoted as $N(S)$. Partial transformation semigroups are known to contain empty transformation as such they contain nilpotent elements. Laradji and Umar (2004) found the number of nilpotent elements in $P_{n}$ to be:

$$
\left|N\left(P_{n}\right)\right|=\left(\begin{array}{l}
n \\
r
\end{array}\right) \mathrm{S}(\mathrm{n}, \mathrm{r}+1) \mathrm{r} !=(n+1)^{n-1} .
$$

Question(3) Now if $S=C P_{n}$, what is $|N(S)|$ ? This remain an open problem.

\section{The semigroup of full transformations}

Recall that $\alpha$ is said to be a full transformation if $\operatorname{Dom} \alpha=[n]$ and the collection of all full transformations of [n] is known to be the semigroup of full(or total) transformation usually denoted by $T_{n}$. This semigroup has also been extensively studied in the literature just like $P_{n}$. In this section we itemize some of the well know combinatorial properties of this semigroup and we ask a similar question for its subsemigroup of full contractions $C T_{n}$. We begin with the following lemmas.

Lemma3.2 ([10]) Let $S=P_{n}$. Then $F(n ; p)=$ $\left(\begin{array}{l}n \\ p\end{array}\right) S(n, p) p !,(n \geq p \geq 1)$.
Lemma3.3 ([12], Corollary 2.6) Let $=P_{n}$. Then $F(n ; m)=\left(\begin{array}{l}n \\ m\end{array}\right)(n-1)^{n-m},(n \geq m \geq 0)$.

Lemma 3.4 ([12], Corollary 2.9) Let $=P_{n}$. Then $F(n ; k)=(k)^{n}-(k-1)^{n},(n \geq k \geq 1)$.

We also pause here to elaborate on the above lemmas, these lemmas have solve the problems of a 2-parameter functions for the semigroup of full transformations $T_{n}$. And summation over the any of the parameter gives the order of the semigroup. The order of $T_{n}$ is known to be $n^{n}$. Thus, we ask a similar question as follows:

Question (4) What is $(n ; r), F(n ; p), \quad F(n ; m)$ and $F(n ; k)$ when we replace $T_{n}$ with $C T_{n}$ ? These questions remain an open problem. What is the order of this semigroup?

Similarly the number of idempotent in $T_{n}$ as found in [3] is given as:

$$
|E(S)|=\sum_{r=1}^{n}\left(\begin{array}{l}
n \\
r
\end{array}\right)(r)^{n-r} .
$$

Question (5) Now what if $S=C T_{n}$ ? What is $|E(S)|$ ? This question remain an open problem.

Remark (6) Notice that since for all $\alpha$ in $T_{n}$, Dom $\alpha=[n] \neq\{\}$ then $T_{n}$ has no nilpotent.

Regularity of elements in $D C P_{n}$ and $D C T_{n}$. Now recall that a transformation $\alpha \in C P_{n}$ (resp., $\alpha \in C T_{n}$ ) is said to be order decreasing if $x \alpha \leq x$ for all $x \in \operatorname{Dom} \alpha$ (resp., $x \in[n]$ ). An element $\alpha$ in a semigroup $S$ is said to be regular if and only if there exists $\beta$ in $S$ such that $\alpha=\alpha \beta \alpha$. If all elements of $S$ are regular then $S$ is said to be a regular semigroup. Many transformation semigroups were shown to be regular or their regular elements are characterized. For example in 2018 the semigroup $C P_{n}$ was shown to be non regular and its regular elements have been characterized (Ali et al., 2018).

Let $\alpha=\left(\begin{array}{ccc}A_{1} & \cdots & A_{1} \\ & & \\ x_{1} & \cdots & x_{r}\end{array}\right)$

The collection $T_{\alpha}=\left\{t_{i} \in A_{i}: 1 \leq i \leq r\right\}$ with $\left|T_{\alpha}\right|=r$ is called a transversal. A transversal $T_{\alpha}$ is said to be admissible if the map $\left(\begin{array}{ccc}A_{1} & \cdots & A_{1} \\ & & \\ t_{1} & \cdots & t_{r}\end{array}\right)$ is a contraction. For proper concept of admissibility see (Ali et al., 2018). We now give the following result from Ali et. al which we shall need in the subsequent results.

Theorem 4.1 (Ali et al., 2018), Theorem 2.1) Let $\alpha \in C P_{n}$ be as expressed in equation (1). Then $\alpha$ is regular if and only if there exists an admissible transversal $T_{\alpha}$ of $\operatorname{Ker} \alpha$ such that $\left|t_{j}-t_{i}\right|=\left|t_{j} \alpha-t_{i} \alpha\right|$ for all $t_{j}, t_{i} \in T_{\alpha} \quad(i, j \in$ $\{1,2, \ldots r\})$.

We now characterize the regular elements in $D C P_{n}$ and $D C T_{n}$. 
Remark 4.2 The above results is the same when we replace $C P_{n}$ with $D C P_{n}$.

Before we give the characterization of regular elements of $D C T_{n}$, we first give the following definition. A transversal $T_{\alpha}=\left\{a_{1}, a_{2}, \ldots, a_{r}\right\}$ is said to be upper if $a_{1} \geq a_{2} \geq \cdots \geq a_{r}$ and is lower if $a_{1} \leq a_{2} \leq \cdots \leq a_{r}$.

Now we deduce from Theorem 4.1 the following Lemma.

Lemma 4.3 Let $\alpha \in D C T_{n}$ be as expressed in equation (1). Then $\alpha$ is regular if and only if there exists a lower admissible transversal $T_{\alpha}$ of Ker $\alpha$ such that $\left|t_{j}-t_{i}\right|=\left|t_{j} \alpha-t_{i} \alpha\right|$ for all $t_{j}, t_{i} \in T_{\alpha}(i, j \in\{1,2, \ldots r\})$.

Proof. The result follows from Theorem 4.1 and the fact that every admissible transversal is necessary lower since $\alpha$ is order decreasing.

Corollary 4.4 The semigroup $D C T_{n}$ is not regular.

Let $F(n, r)=\left|\left\{\alpha \in D C T_{n}:|i m \alpha|=r\right\}\right|$. Then we have the following trivial results.

Lemma 4.4 If $S=D C T_{n}$ then $F(n, r)=$ S1 if $r=1$

$\{1$ if $r=n$

proof:

Notice that $\left\{\alpha \in D C T_{n}:|i m \alpha|=1\right\}=\left\{\left(\begin{array}{c}{[n]} \\ 1\end{array}\right)\right\}$ and $\left\{\alpha \in D C T_{n}:|\operatorname{im} \alpha|=n\right\}=\left\{\left(\begin{array}{ccc}1 & \cdots & n \\ & & \\ 1 & \cdots & n\end{array}\right)\right\}$.

\section{REFERENCES}

Ali, B., Umar, A. and Zubairu, M. M. (2018). Regularity and Green's relations on the semigroup of partial contractions of a finite chain. arXiv:1803.02146v1.

Adeshola, A. D. and Umar, A. (2013). Combinatorial results for certain semigroups of order- preserving full contraction mappings of a finite chain. J. Comb. Maths. and Comb. Computing

Clifford, A. H. and Preston, G.B. (1961). The algebraic theory of semigroups, vol.1. Providence, R. I.: American Mathematical Society,.

Garba, G. U. (1990). Nilpotents in partial one-toone order-preserving transformations, Semigroup Forum 116 A., 359-366.

Garba, G. U., Ibrahim, M. J. and Imam, A. T. (2017). On certain semigroups of full contraction maps of a finite chain. Turk. J. Math. 41: 500-507.

Garba, G. U. (1990). Idempotent in partial transformation semigroup. Proc. Roy. Soc. Edinburgh. 116A; 359-366.

Ganyushkin, O. and Mazorchuk, V. (2009). Classical Finite Transformation Semigroups. Springer Verlag: London Limited.

Gomes, G. M. S. and Howie, J. M., (1987). Nilpotents in finite symmetric inverse semigroups, Proc. Edinburgh Math. Soc. 30, 383-395.
As such the result follows.

We conclude the paper with the following conjuncture;

Conjuncture 4.5 If $S=D C T_{n}$ then $F(n, r)=$ $\left\{2^{n-1}-1 ; \quad\right.$ if $r=2$

$\{2(\mathrm{n}-3)(\mathrm{n}-2) ;$ if $r=3$

\section{CONCLUSION}

All these combinatorial functions when computed gives rise to some sequence or triangle of numbers. This numbers are fascinating and solves allot of problems in algorithm, graph theory, automata, e .t. c.

\section{Recommendation}

Due to the importance of Combinatorics which arises often from the study of finite transformation semigroups in science and technology, there is need to solve these combinatorial problems, as such we recommend the study of these combinatorial problems, which we hope it will explore some new (or existing) triangle of numbers or sequences.

Contribution of individual authors

Muhammad Mansur Zubairu was the PhD student who characterized the regular elements and both authors itemized the combinatorial questions.

Howie, J. M.(1995). Fundamental of semigroup theory. London Mathematical Society, New series 12. The Clarendon Press, Oxford University Press,

Howie, J. M. and Mcfadden, R. B. (1990). Idempotents rank in finite full transformations semigroups, Proc. Roy. Soc. Edinburgh. 114A, 161-167.

Laradji, A. and Umar, A. (2004). On the number of nilpotents in the partial symmetric semigroup. Comm. Algebra 32(8), 3017\{3023.

Umar, A. (2014). Some combinatorial problems in the theory of partial transformation semigroups. Journal of Algebra and Discrete Mathematics 17 1:110\{134.

Umar, A. and Zubairu, M. M. (2018). On certain semigroups of partial contractions of a finite chain. arXiv:1803.02604.

Umar, A. and Zubairu, M. M. (2018). On certain semigroups of full contractions of a finite chain. arXiv:1804.10057.

Umar, A. and Al-Kharousi, F. (2012). Studies in semigroup of contraction mappings of a finite chain. The Research Council of Oman Research grant proposal No. ORG/CBS/12/007,

Zhao, P. and Yang, M. (2012). Regularity and Green's relations on semigroups of transformation preserving order and compression. Bull. Korean Math. Soc. 49, No. 5, 1015-1025. 